\title{
Esfera pública y música: claves introductorias
}

Quien traduce un texto es invisible. Su labor pasa inadvertida para los lectores, salvo si estos encuentran una expresión poco idiomática, una frase de difícil comprensión o, caso más raro, si conocen la versión original del texto y esta no se corresponde con la versión traducida que tienen ante sus ojos. Por todo ello, resulta aún más extraordinario el alto grado de visibilidad alcanzado por Antoni Domènech como traductor al castellano -con la colaboración de Rafael Grasa- del texto de Jürgen Habermas Strukturwandel der Öffentlichkeit. Untersuchungen zu einer Kategorie der bürgerlichen Gesellschaft, punto de partida teórico del dosier introducido por este artículo ${ }^{1}$.

En una extensa nota al comienzo del libro, Domènech expresa su profundo desacuerdo con el criterio de la editorial a la hora de decidir el título. La traducción literal del original habermasiano sería: El cambio estructural de la publicidad. Investigaciones sobre una categoría de la sociedad burguesa. La clave (y la dificultad) de este título reside en el término "publicidad", traducción del alemán Öffentlichkeit. Comoquiera que, en castellano moderno, el término "publicidad" ha perdido el significado de "vida social pública" que tenía antiguamente, el traductor propuso titular el libro La transformación estructural de la vida pública, aceptando el sintagma "vida pública" como traducción actualizada de "publicidad", y reservando este último término para las páginas interiores. Sin embargo, la editorial decidió que Historia y crítica de la opinión pública. La transformación estructural de la vida pública sería un título más adecuado. De este modo, se enfatizaban los vínculos de la propuesta habermasiana con la teoría de la comunicación (después de todo, el libro se publicaba dentro de una serie sobre comunicación y medios de comunicación). Pero, sobre todo -y esta es una cuestión que Domènech parece pasar por alto-, se eliminaba toda referencia al carácter burgués de la esfera pública estudiada por $\mathrm{Ha}-$ bermas. Dicho de otro modo: se disimulaba la raíz marxista del pensamiento habermasiano en los años previos al advenimiento del "fin de la historia".

Las líneas anteriores ponen de manifiesto tres elementos que han marcado la difusión, la recepción y la asimilación del pensamiento habermasiano. En primer lugar, la complejidad de los conceptos manejados por el filósofo. En segundo lugar, la incidencia del contexto ideológico en la

\footnotetext{
${ }^{1}$ En el momento de revisar este texto conozco el inesperado fallecimiento de Antoni Domènech. Sirvan estas líneas como reconocimiento a su figura.
} 
recepción de su pensamiento.Y, finalmente, las dificultades inherentes a la traducción del texto.Todas estas cuestiones permiten explicar, aunque solo en parte, su parsimoniosa difusión internacional, su tardía asimilación en el campo de la musicología, y su (aún) más tardía asimilación por parte de las musicologías española y latinoamericana.

En las próximas páginas se ofrece una somera síntesis del proceso de difusión y recepción de Historia y crítica... y se presentan los conceptos fundamentales del texto de Habermas, algunas de las críticas al mismo y algunas de las visiones que, partiendo de su modelo, lo amplían o redefinen. De este modo, se aspira a ofrecer una introducción conceptual a los artículos que forman el presente dosier.

\section{Strukturwandel der Öffentlichkeit: génesis y difusión de un texto}

Cuando Jürgen Habermas comienza a escribir Historia y crítica de la opinión pública aún queda más de un lustro para mayo del 68. El texto, que se adelanta a muchas de las tesis de la nueva izquierda sesentayochista (la crítica a los medios de comunicación de masas, a la burocracia, a los partidos institucionalizados, a la comercialización de la cultura...), había surgido en 1960. Fue entonces cuando el joven filósofo y sociólogo decidió redactar su tesis de habilitación en Marburgo bajo la dirección del jurista y politólogo socialdemócrata Wolfgang Abendroth. Previamente había trabajado con Theodor W. Adorno y Max Horkheimer en el Instituto de Investigación Social de Fráncfort, donde estaba previsto inicialmente que defendiera esta tesis. Sin embargo, Horkheimer y Adorno podrían haber encontrado el enfoque habermasiano demasiado poco crítico con la concepción ilustrada de la vida pública democrática. Por ello, el pensador se desvinculó orgánicamente de la Escuela de Fráncfort, de la que, por otra parte, es considerado un continuador ${ }^{2}$.

Historia y crítica salió de la imprenta en 1962. El énfasis en cuestiones sociológicas y politológicas de ulteriores trabajos de Habermas (quien regresó a Fráncfort entre 1964 y 1971 para ejercer como catedrático en la universidad) llamaron pronto la atención de los críticos y comentadores de su obra, que soslayaron la importancia de este trabajo ${ }^{3}$. Sin embargo, los sucesos del 68 y el giro cultural experimentado en años posteriores por las humanidades y las ciencias sociales despertaron el interés por el texto y favorecieron su difusión en todo el mundo.

\footnotetext{
${ }^{2}$ A. Domènech: "Prólogo a la edición castellana: El diagnóstico de Jürgen Habermas, veinte años después", Jürgen Habermas: Historia y crítica de la opinión pública. La transformación estructural de la vida pública, Barcelona, G. Gili, 1981. Traducción de Antoni Domènech y Rafael Grasa, p. 14; Craig J. Calhoun: "Introduction: Habermas and the Public Sphere", Habermas and the Public Sphere, Craig J. Calhoun (ed.), Cambridge, Massachussets, MIT Press, 1992, pp. 1-47.

3 Juan Carlos Domènech Velasco: Para leer a Habermas, Madrid, Alianza, 2003.
} 
Hasta donde sabemos, la primera traducción de Historia y crítica fue la italiana, de 1971. Su título, Storia e critica dell'opinione pubblica ${ }^{4}$, guarda un evidente parecido con el elegido por los editores españoles, al tiempo que la participación de un equipo interdisciplinar resulta reveladora de las exigencias del original habermasiano. A partir de ahí se sucederían las traducciones al francés ${ }^{5}$ (1978, por el filósofo y traductor Marc de Launay), al castellano (1981), al portugués ${ }^{6}$ (1984) y, finalmente, al inglés ${ }^{7}$ (1989).

Un somero análisis de esta sucesión de traducciones revela hasta qué punto el advenimiento de la posmodernidad actuó como catalizador de la difusión de Historia y crítica. Con todo, la recepción del texto se vio influida por las circunstancias particulares del lugar y del momento en que se produce cada traducción. Por ejemplo, en el caso español, el estudio introductorio de Antoni Domènech aparece marcado por la desilusión y el desencanto. Esta actitud es consecuencia del triunfo de la visión radical y desacomplejada del capitalismo sostenida por un Reagan que acababa de ganar las elecciones estadounidenses, y que parecía encarnar una de las más pesimistas profecías habermasianas: la disolución del debate racional en la esfera pública, suplantado por las técnicas de la "publicidad" en el sentido moderno del término; el triunfo de la demagogia.

Por su parte, en su introducción a la versión inglesa, el filósofo Thomas McCarthy (uno de los mayores expertos en Habermas del área anglosajona) reconoce el injustificable retraso en la traducción del texto dirigida al público anglosajón. Pero, sobre todo, señala cómo las corrientes intelectuales del momento en que se produce parecen predisponer a una buena acogida del mismo $^{8}$. En efecto, las transformaciones disciplinarias en las ciencias humanas y sociales, con su renovado énfasis en los aspectos sociológicos y culturales, auguraban una buena acogida de Historia y crítica, como de hecho sucedió. A decir verdad, el ámbito anglosajón ya había tenido acceso a la traducción de un artículo de Habermas en el que se sintetizaban los conceptos y aspectos fundamentales sobre el tema9. Además, el propio McCarthy había editado un volumen sobre la filosofía del alemán ${ }^{10}$, y

${ }^{4}$ J. Habermas: Storia e critica dell’opinione pubblica, Bari, Laterza, 1971. Traducción de Augusto Illuminati, Ferruccio Masini y Wanda Perretta.

${ }^{5}$ J. Habermas: L'espace public. Archéologie de la publicité comme dimensión constitutive de la société bourgeoise, París, Payot, 1978. Traducción de Marc de Launay.

${ }^{6} \mathrm{~J}$. Habermas: Mudança estrutural da esfera pública: investigações quanto a uma categoría da sociedade burguesa, Río de Janeiro, Tempo Brasileiro, 1984. Traducción de Flávio R. Kothe.

${ }^{7}$ J. Habermas: The Structural Transformation of the Public Sphere. An Inquiry into a Category of Bourgeois Society, Cambridge, Massachussets, 1989. Traducción de Thomas Burger y Frederick Lawrence.

${ }^{8}$ T. McCharthy: "Introduction", pp. XI-XVI.

9 J. Habermas: "The Public Sphere: An Encyclopedia Article (1964)", New German Critique, n. o 3, 1974, pp. 49-55. Traducción de Sara Lennox y Frank Lennox.

${ }_{10}$ T. McCarthy: The Critical Theory of Jürgen Habermas, Cambridge, Massachussets, MIT, 1978. 
pronto proliferaron textos que analizaban, rebatían, repensaban y aplicaban a los más diversos campos el concepto de la esfera pública ${ }^{11}$. La posmodernidad académica se hallaba en una fase madura y todas las disciplinas se aprestaban a realizar su giro cultural. En ese contexto, la visión habermasiana podía resultar una poderosa herramienta de análisis, como continúa siendo hasta la actualidad.

\section{La esfera pública burguesa ${ }^{12}$}

El concepto de "esfera pública" (Öffentlichkeit), que Habermas complementa con el marchamo de "burguesa", constituye el núcleo de su trabajo y es, asimismo, el elemento aglutinador de los artículos que forman este dosier. Por ello, conviene comenzar señalando que la esfera pública es un término histórico vinculado a una clase social determinada que cabe definir como el lugar conceptual donde "las personas privadas se reúnen en calidad de público"13 (es decir, donde hacen uso público de su razón).

El punto de partida del concepto es la distinción entre el ámbito privado y el ámbito público, una distinción que no aparecería hasta el siglo XVIII, con el desarrollo de las estructuras que dieron lugar al sistema capitalista. Anteriormente, en el feudalismo y en el absolutismo, quien ejercía el poder desde el ámbito político o religioso expresaba su estatus de manera concreta y directa. Los monarcas, los nobles o las autoridades religiosas representaban (encarnaban, hacían visible) el poder ante el pueblo, y no por delegación o en representación de este. Por ello, Habermas denomina "publicidad representativa" a la vida social característica de aquella época, que encuentra su ejemplo más acabado en la figura de Luis XIV y en el complejo ceremonial que le rodeaba, con el dormitorio del monarca-que hoy no dudaríamos en identificar con el espacio más privado de una casacomo epicentro del aparato representativo de la corte ${ }^{14}$.

La situación se transformaría radicalmente con el desarrollo del capitalismo financiero y comercial, que lleva a distinguir con claridad entre los aspectos privado y público de la vida. Desde finales del siglo XVII, y a lo largo del XVIII, el avance del comercio obliga a las instituciones tradicionales a redefinir su campo de acción y, sobre todo, permite a la burguesía articular un espacio de diálogo racional y crítico, ajeno a la autoridad pública y a las actividades de la vida privada de los individuos. La esfera

\footnotetext{
${ }^{11}$ Entre ellos cabe destacar Craig J. Calhoun (ed.): Habermas...

12 Frente al término "publicidad", utilizado por Domènech en su traducción, utilizaré el término "esfera pública" por resultar menos equívoco para el lector actual, así como por tratarse del término más extendido, sin duda por influencia anglosajona, para referirse en castellano a la Öffentlichkeit.

${ }^{13} \mathrm{~J}$. Habermas: Historia y crítica..., p. 65.

${ }_{14}$ Ibid., pp. 44-51.
} 
pública burguesa es, por tanto, el ámbito en el que las personas privadas, excluidas del ejercicio del poder político, se reúnen y hacen valer sus intereses formando un cuerpo público, ajeno al Estado y -de acuerdo con Habermas- crítico con los fundamentos de este ${ }^{15}$. Al hacerlo, construyen un espacio en el que se establecen relaciones que median entre los ámbitos de la sociedad civil y el Estado ${ }^{16}$.

La esfera pública es un espacio homogéneo, no compartimentado (es decir, no jerarquizado), en el que la razón no es limitada por poderes religiosos o políticos. Todas las personas libres y capaces de acceder a la cultura pueden participar de ella, intervenir en los debates y, en definitiva, hacer un uso público de su razón. Habermas examina, además, cuáles son los espacios físicos en los que se construye la esfera pública. En su visión, dado que la burguesía no puede acceder al poder político, se ve impelida a desarrollar una serie de instituciones de sociabilidad en las que podrá desarrollar esta esfera pública libre y con las que expandirá su visión cultural: los clubes, los salones, las logias masónicas, los cafés, los teatros y, de manera particular, los conciertos públicos ${ }^{17}$.

En todos estos espacios, situados al margen del Estado y gobernados por el debate racional, fue posible articular lugares de encuentro al margen de clases sociales. Pero, además, es allí donde la cultura pierde su carácter representativo y llega a convertirse en un bien de consumo: los objetos artísticos pueden ahora estar desprovistos de "significado" y ser apreciados por su propia naturaleza; pueden ser comprados, vendidos e intercambiados, y, por eso mismo, pueden ser criticados sobre fundamentos racionales. Es así como la noción habermasiana de la esfera pública permite explicar de manera integral el nacimiento de la estética y de la crítica artística, así como el desarrollo de determinados géneros y prácticas musicales, abarcando a la vez aspectos económicos, políticos, sociales y filosóficos.

Los espacios de socialización antes mencionados constituyen el entorno en el que se constituye el "público"; un público eminentemente burgués, cuyo mayor grado de influencia sitúa Habermas en torno a $1750^{18}$. La formación de este público se produce en capas, desde el ámbito privado hasta el público: la consolidación del núcleo familiar patriarcal como modelo de estructura familiar burguesa (frente a la "casa" familiar aristocrática, en la que rigen grados de parentesco tanto sanguíneo como laboral) es el primer paso hacia la diferenciación entre espacio público y espacio privado, hacia una distinción entre la vida familiar íntima y la actividad económica que no existía

\footnotetext{
${ }^{15}$ Ibid., p. 65. Véase también J. Habermas: "The Public Sphere...", pp. 51-52.

16 T. McCarthy: The Critical Theory..., p. 381

17 J. Habermas: Historia y crítica..., pp. 69.

18 Ibid., p. 81.
} 
en siglos anteriores. La socialización de estos individuos burgueses en los espacios físicos mencionados (cafés, museos, bibliotecas, conciertos), o a través de medios impresos, constituye, por tanto, el germen del desarrollo del "público" (o de los públicos), entendido como una comunidad de individuos privados que se expresan racionalmente en la esfera pública.

Es preciso advertir que la constitución de un público de individuos privados con capacidad para emitir juicios críticos supone, al mismo tiempo, una ampliación y una exclusión con respecto a las instituciones que ostentaban tradicionalmente el monopolio de la crítica (la corte, las academias, los expertos). Ampliación, porque esta comunidad crítica da cabida, en principio, a todas las personas privadas que, como lectores, oyentes y espectadores, eran capaces de dominar los temas de discusión. Exclusión, porque no todos los individuos pueden tener acceso a los bienes y a la cultura, y porque el debate político no estaba al alcance de una mayoría de individuos, carentes de los conocimientos que garantizaban la expresión de juicios críticos racionales ${ }^{19}$.

En cualquier caso, el elemento que permitirá solidificar estos públicos será la comunicación escrita. La "revolución lectora", que multiplica las cartas (tanto las que se cruzan entre individuos privados como las que se escriben con destino a la imprenta) y que favorece el desarrollo de la prensa, pone en contacto a personas que comparten opiniones similares, aunque vivan en lugares distantes ${ }^{20}$. La importancia de la comunicación escrita ya había sido reivindicada por autores de la época como Kant. Según el filósofo, este tipo de comunicación permitía el intercambio con quienes estaban ausentes y, sobre todo, permitía generar un espacio para el debate de ideas que dejaba de estar restringido por cuestiones geográficas y podía llegar a ser verdaderamente universal ${ }^{21}$. Por este motivo, la prensa, en tanto que vehículo para la expresión de la crítica y para la creación de opinión, es la institución que se vincula de un modo más claro a la aparición de la esfera pública, y la que permite fortalecer de un modo más poderoso en los individuos privados la conciencia de pertenencia a un público más amplio, interconectado y cohesionado.

Así, mediante su participación en instituciones de socialización burguesa y en publicaciones impresas, los hombres y mujeres cultos que participaban de la esfera pública transformaron el panorama político y social del siglo XVIII, al tiempo que tomaron conciencia y se empoderaron como

\footnotetext{
${ }^{19}$ Roger Chartier: Espacio público, crítica y desacralización en el siglo XVIII. Los orígenes culturales de la Revolución Francesa, Barcelona, Gedisa, 2003, p. 35. Traducción de Beatriz Lonné.

20 Tim C. W. Blanning: The Culture of Power and the Power of Culture. Old Regime Europe 1660-1789, Oxford, Oxford University Press, 2002, pp. 132-135.

${ }^{21}$ R. Chartier: Espacio público, crítica y desacralización..., pp. 36-40. Esta reivindicación kantiana se comprende aún mejor si se atiende a la psicología y la biografía del filósofo, hombre que apenas salió de su Königsberg natal, pero que estaba informado de las noticias que sucedían en toda Europa.
} 
individuos autónomos ${ }^{22}$. Desde ese momento, esos individuos, constituidos en público, llegan a erigirse en portadores de la "opinión pública”, entendida como la expresión racional de la minoría que es capaz de emitir juicios críticos públicamente. Pero, como ha analizado Roger Chartier, la opinión pública es vista a lo largo del siglo XVIII como lo opuesto a la opinión popular; el público es confrontado con el pueblo y, si aquel se expresa de manera estable, única y racional, este lo hace en función de prejuicios y pasiones, generando una opinión que es múltiple y variable. Por ello, resulta siempre manipulable y no puede ser considerado un sujeto político, sino que debe ser guiado de forma casi paternalista ${ }^{23}$. El modelo habermasiano beberá de estas fuentes y llegará a considerar como una amenaza para la esfera pública ilustrada la irrupción en ella de las masas iletradas.

En efecto, el modelo ideal de la esfera pública habermasiana se circunscribe al siglo XVIII. Solo en el momento previo a la institución del Estado liberal podía desarrollarse ese terreno de debate racional en el que los individuos privados podían formular públicamente sus opiniones en busca del bien común. El desarrollo del capitalismo y de las estructuras estatales asociadas a él conduce al definitivo triunfo de la burguesía, que no solo consigue imponer su visión sobre la realidad, sino que alcanza el poder político. Pero, al mismo tiempo, este triunfo significa dar acceso a la esfera pública a las masas desposeídas e ignorantes, que no están capacitadas para realizar un análisis racional de la realidad y que amenazan el orden político-social instaurado por la burguesía.

Con esta irrupción, la esfera pública entraría en una nueva etapa, en la que se esfumaría toda posibilidad de establecer un gobierno y una legislación racionales; en la que la política podría caer fácilmente por el precipicio de la demagogia, y en la que el periodismo abandona su función como difusor de noticias y se convierte en un intensificador de la discusión pública. El progresivo afianzamiento de las estructuras del capitalismo conduciría a la aparición de un público consumidor de la industria cultural, y a la desaparición de una esfera pública racional, sustituida por un cúmulo de instituciones que cumplen con la función de mediar entre la sociedad civil y el Estado.

\section{La expansión del modelo habermasiano}

El panorama histórico relatado no puede ser más desolador. En él se refleja con claridad un aristocracismo cultural, heredero de la Escuela de Fráncfort, que lleva a identificar la irrupción de las masas iletradas con la

22 Deena Goodman: "The public and the nation", Eighteenth Century Studies, vol. 29, n. ${ }^{\circ}$ 1, 1995, p. 1.

${ }^{23}$ R. Chartier: Espacio público, crítica y desacralización..., pp. 40-43. 
ruina de los ideales ilustrados ${ }^{24}$. En los ojos de Habermas, por tanto, la esfera pública será burguesa o no será. Pero esta naturaleza burguesa de la esfera pública resulta escurridiza, en primer lugar, porque el término alemán bürgerlich puede traducirse como "civil" (en el sentido de "sociedad civil"), "doméstico" (en el sentido de la tragedia doméstica, o burguesa, del siglo XVIII),"aburguesado", además de "burgués" (perteneciente a la clase social burguesa) ${ }^{25}$.

La utilización del término "burgués" en un sentido social lleva a enraizar la noción de esfera pública en la identidad de una clase determinada. Se trata, por tanto, de una lectura esencialmente marxista que, sin embargo, será sometida a crítica desde fechas tempranas. El propio Antoni Domènech, incardinado en la tradición marxista, apunta a la debilidad de esta visión al tiempo que defiende la existencia de una "sólida y robusta publicidad plebeya [...] o proletaria", de la que se apercibió Karl Marx, pero que acabaría parcialmente destruida con la industrialización y el desarrollo capitalistas ${ }^{26}$.

Desde otros ámbitos, autores como Tim Blanning han defendido el carácter diverso de la esfera pública, que han definido como un espacio socialmente heterogéneo y políticamente multidireccional ${ }^{27}$. Desde el punto de vista social, la heterogeneidad de la esfera pública derivaría de la implicación, no tanto de los elementos plebeyos a los que se refería Domènech, como de un buen número de nobles y religiosos que, en la visión ideal habermasiana, deberían haber quedado al margen. En este sentido, la intervención en la esfera pública vendría marcada, primero, por la capacidad de acceso a la cultura, y, segundo, por el mérito intelectual de cada individuo. La diversidad política de la esfera pública es igualmente reivindicada por el autor británico: frente a la caracterización habermasiana de la esfera pública como un espacio de confrontación con el Estado, Blanning constata la multiplicidad de opiniones políticas coexistentes en ella, al tiempo que recuerda cómo, en numerosas partes de Europa, se establecieron relaciones simbióticas entre los poderes estatales y la esfera pública ${ }^{28}$. De este modo, la esfera pública resultaría de la coexistencia de varias fuerzas (desde la construcción de las instituciones del Estado hasta la aceleración en el intercambio de información) y, en palabras del autor, vendría a parecerse más al Arca de Noé que a un buque mercante ${ }^{29}$.

La naturaleza heterogénea de la esfera pública había sido ya advertida

\footnotetext{
${ }^{24}$ A. Domènech: "Prólogo...", pp. 22-24.

25 T. C. W. Blanning: The Culture of Power..., p. 11-12.

${ }^{26}$ A. Domènech: "Prólogo...", pp. 22-23.

27 T. C. W. Blanning, The Culture of Power..., p. 12.

${ }^{28}$ Ibid., p. 13.

${ }^{29}$ Ibid., p. 12.
} 
desde el ámbito de los estudios culturales y de género por autoras como Joan Landes o Nancy Fraser ${ }^{30}$. Esta última considera que el modelo propuesto por Habermas, en el que se priman los elementos homogeneizadores, resulta excluyente en términos de clase y de género. Por ello, plantea la existencia de "contrapúblicos subalternos", que define como espacios discursivos paralelos donde los miembros de los grupos sociales subordinados pueden construir y difundir contradiscursos ${ }^{31}$. Así, frente a una esfera pública monolítica, existirían una multitud de esferas paralelas en las que diversos públicos articularían discursos múltiples y a menudo contradictorios, pero al mismo tiempo enriquecedores desde el punto de vista democrático.

La última de las principales críticas lanzadas al modelo habermasiano es de índole cronológica. De acuerdo con su narración histórica, la discusión sobre cuestiones culturales, artísticas y literarias antecedió al debate político y sirvió para que los participantes en la esfera pública entrenaran sus habilidades dialécticas. Sin embargo, existen ejemplos que contradicen esta asunción, como son los casos inglés y francés, donde el debate sobre cuestiones políticas en el siglo XVII se produjo casi cien años antes de que se articulara un debate similar en términos artísticos ${ }^{32}$.

En cualquier caso, a pesar de las críticas y reelaboraciones de su teoría, el marco general descrito por Habermas se mantiene firme y resulta útil como herramienta integral de análisis cultural, social y político, particularmente atractivo en el ámbito musicológico. Y no resulta extraño que la musicología pueda sacar provecho del marco teórico ofrecido por Habermas, habida cuenta de la importancia que el propio autor atribuye a la actividad musical, y en particular al concierto público, en el desarrollo de la esfera pública:

La transformación habida puede observarse aún más claramente en el público de conciertos que en el público lector o espectador; esa transformación no ha acarreado cambio en el público, sino que ha dado lugar al "público" mismo como tal. [...] La entrada de pago convirtió al concierto en mercancía; al mismo tiempo, apareció algo así como una música desvinculada de fines u obligaciones: por vez primera se juntaba un público a oír música como tal; un público amante de la música, al que se le suponen patrimonio e instrucción, ha entrado en escena ${ }^{33}$.

\footnotetext{
30 Joan Landes: Women and the Public Sphere in the Age of the French Revolution, Ithaka, Cornell University Press, 1988; Nancy Fraser: "Rethinking the Public Sphere: A Contribution to the Critique of Actually Existing Democracy", C. Calhoun: Habermas..., pp. 109-142.

${ }^{31}$ La noción de "contrapúblico" ha sido reivindicada por Michael Warner, quien la utiliza en su intento de responder a la pregunta "qué es un público". Michael Warner: Publics and Counterpublics, Cambridge, Massachussets, MIT, 2002.

32 T. C. W. Blanning: The Culture of Power..., pp. 13-14.

33 J. Habermas: Historia y crítica..., pp. 76-77.
} 
En estas palabras, y en las referencias bibliográficas manejadas por Habermas, se hace patente la influencia que sobre su concepción de la esfera pública ejerció la tradición sociológica y, sobre todo, los estudios vinculados a la Escuela de Fráncfort, más allá de Adorno. En concreto, resultan notables sus referencias al volumen colectivo sobre el aburguesamiento del arte, la literatura y la música alemanes del siglo XVIII, firmado por Gert Mattenkott, Ernst Rebling y Leo Balet y publicado en $1936^{34}$. Este trabajo constituye un hito en el desarrollo de una historia social de la música, y enlaza con la segunda referencia más notable de entre las citadas por Habermas: la que remite al historiador del arte húngaro Arnold Hauser, autor de la conocida Historia social de la literatura y el arte (1951) y de la no menos conocida Sociología del arte (1975).

La sociología del arte, que había florecido en los años de la República de Weimar ${ }^{35} \mathrm{y}$ se había prolongado en ciertos trabajos de la posguerra, nutre, por tanto, la visión habermasiana del hecho musical. De este modo, en Teoría y crítica se enfatizan los vínculos que unen la práctica artística y el sustrato social, con una perspectiva que tardaría en llegar al discurso musicológico predominante, por mucho tiempo aferrado en exclusiva a la tradición positivista. En todo caso, y de manera progresiva, nociones como la de "espacio público" (más que la de "esfera pública") comenzaron a interesar a los practicantes de la musicología urbana, quienes encontraron útil este concepto para subrayar el aspecto topográfico, material y tangible, de la esfera pública. Se hacía posible así trazar una línea divisoria entre la actividad musical privada de tipo institucional o comercial (la música sacra, la ópera o incluso el concierto público) y aquellas manifestaciones sonoras que tienen lugar "de puertas afuera" en la ciudad, es decir, en el marco de una geografia cívica, medial y neutral (es decir, pública). Al mismo tiempo, en los últimos años se han sucedido los estudios focalizados en las prácticas musicales domésticas (es decir, las prácticas privadas por antonomasia), para las que se producían o adaptaban unos repertorios musicales concretos, distintos de los interpretados en las salas de conciertos ${ }^{36}$.

${ }^{34}$ Gert Mattenkott, Ernst Rebling y Leo Balet: Die Verbürgerlichung der Deutschen Kunst, Literatur und Musik im 18. Jahrhundert, Estrasburgo y Leiden, Heitz, 1936. En la primera edición, Rebling, que aún no se había exiliado, utilizó el seudónimo de E. Gerhard para evitar represalias políticas.

35 Trabajos pioneros y destacados en esta corriente se deben a Adorno y a Max Weber (Los fundamentos racionales y sociológicos de la música, de Max Weber, 1921). En la misma línea habían trabajado, en el Berlín de Weimar y bajo un prisma claramente marxista que aunaba teoría y práctica, autores como Fritz Jöde y Hans Boettcher. Véase: Elizabeth Janki: Recomposing German Music: Politics and Musical Tradition in Cold War Berlin, Leiden, Brill, 2005, pp. 54-55.

${ }^{36}$ A modo de ejemplo, pueden verse, Mary Hunter: "Haydn's London Piano Trios and His Salomon String Quartets: Private vs. Public?", Haydn and His World, Elaine Sisman (ed.), Princeton, Princeton University Press, 1998, pp. 103-130; Marie Sumner Lott: The Social Worlds of Nineteenth-Century Chamber Music. Composers, consumers, communities, Chicago, University of Illinois Press, 2015. 
Por otra parte, es de recordar la importancia que Habermas atribuye a la palabra impresa en la configuración del público o de los públicos. En este sentido, no resulta extraño que la musicología haya dejado de ver la crítica musical como un mero recurso al que acudir para buscar fechas o datos sobre el estreno de tal o cual obra. Ahora, la crítica permite (re)conocer comunidades de oyentes, estudiar el proceso de formación de los públicos y, en muchos casos, la manera en que estas dinámicas se incardinan en procesos de construcción nacional. Esta última cuestión no es baladí, habida cuenta de que el nacionalismo constituye un importante agente movilizador del discurso público, particularmente en aquellos momentos en los que existe una crisis de legitimación derivada de la desaparición de viejas formas de autoridad. Es fácil encontrar ejemplos en la Francia posrevolucionaria, en la España del Sexenio Democrático o en la España posnoventayochista de esa fusión entre crítica musical y discurso nacional a la que nos venimos refiriendo.

Quien escribe estas líneas es consciente de la escasez de reflexiones metodológicas en el marco musicológico iberoamericano y, al mismo tiempo, está convencido de que el concepto de esfera pública abre un enorme campo de exploración que puede beneficiar a nuestra disciplina. Por ello, el presente dosier -que inaugura esta nueva sección de los Cuadernos de Música Iberoamericana- presenta tres artículos en los que se unen la reflexión teórica y el análisis práctico. Con este planteamiento se aspira a reforzar el conocimiento de este modelo de análisis y a mostrar diversos ejemplos de los resultados que es posible alcanzar mediante su utilización.

Centrados en el ámbito español (y, en particular, madrileño), estos tres artículos cubren un arco cronológico de más de un siglo y abordan diversas facetas de la relación entre música y la esfera pública. La importancia de este arte en la configuración de la vida social pública aparece ya en el siglo XVIII, en un marco donde coexisten los periódicos (vehículos de expresión del público ilustrado que tiene pleno acceso a la cultura) y las tonadillas (vilipendiados ejemplos de teatro musical en los que -aparentementese expresaba la voz del pueblo). La convivencia y el enfrentamiento entre estos dos medios y su contribución a la formación de la esfera pública es analizada por Ana Sánchez Rojo, quien además plantea cómo el modelo habermasiano funciona, pero solo en parte, para el caso español. Por otra parte, el impulso modernizador vivido por el país durante el reinado de Isabel II permitirá asentar modelos de familia, de socialización y de convivencia característicos del Estado liberal. Como es típico en este tipo de sociedades, las prácticas musicales servirán como aglutinante para unir grupos de individuos que, a su vez, serán la base que configurará las comunidades de oyentes. Con una perspectiva arqueológica, Jesús Cruz Valenciano evalúa en su texto los procesos de formación de distintas comunidades de 
amantes de la música en la España decimonónica, planteando vías de estudio que, a buen seguro, inspirarán futuros trabajos musicológicos. Finalmente, la aparición de un nuevo público musical en la España de la década de 1860 viene a ejemplificar uno de los movimientos a los que Habermas atribuía el fin de la esfera pública burguesa. Como veíamos, la irrupción de una nueva masa social en ámbitos hasta entonces reservados a la burguesía amenaza a las instituciones en las que se desarrollaba el debate racional y, además, conduce a un abaratamiento de la cultura que, convertida en cultura de masas, queda sometida a la dictadura del mercado. Pues bien, el proceso estudiado por Enrique Mejías en torno a la aparición del teatro por horas y su configuración como género dramático-musical encaja con precisión en este marco y permite comprender hasta qué punto se hallan unidas las transformaciones sociales, económicas, políticas y estéticas.

En este punto, solo me resta agradecer al equipo editorial de Cuadernos de música iberoamericana la confianza depositada en mí como coordinador de este dosier, y a Juan José Carreras y Fernando Delgado sus comentarios y correcciones sobre este texto. Asimismo, deseo expresar mi agradecimiento a los revisores de los artículos y, sobre todo, a los autores de los mismos, que se ofrecieron a enviar sus trabajos aun cuando el tiempo jugaba en su contra. 